\title{
Téoros
}

Revue de recherche en tourisme

\section{Un patrimoine de développement régional au profit du tourisme : l'écomusée}

\section{Pierre Mayrand}

Volume 7, numéro 1, mars 1988

Cultures régionales et tourisme

URI : https://id.erudit.org/iderudit/1080434ar

DOI : https://doi.org/10.7202/1080434ar

Aller au sommaire du numéro

Éditeur(s)

Université du Québec à Montréal

ISSN

0712-8657 (imprimé)

1923-2705 (numérique)

Découvrir la revue

Citer cet article

Mayrand, P. (1988). Un patrimoine de développement régional au profit du tourisme : l'écomusée. Téoros, 7(1), 36-37. https://doi.org/10.7202/1080434ar d'utilisation que vous pouvez consulter en ligne.

https://apropos.erudit.org/fr/usagers/politique-dutilisation/ 


\section{Un patrimoine de dévelop- pement régional au profit du tourisme: I'écomusée}

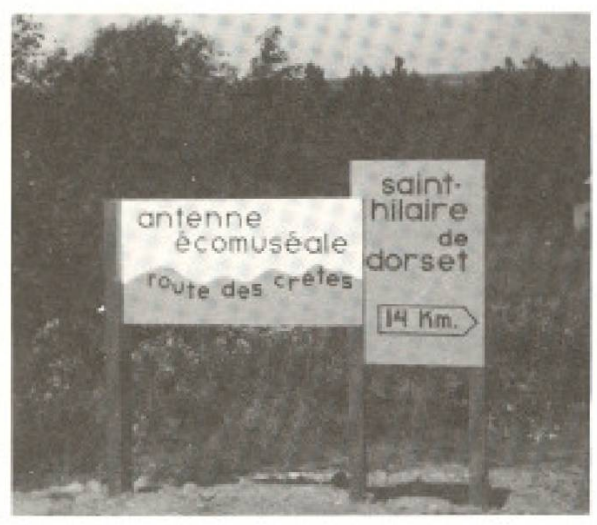

Signalisation routière de l'écomusée. La signalisation est un rappel de la structure de l'exhibit de plein air de Saint Hilaire de Dorset et une référence à la vocation agricole de la région. Elle introduit, en 1981. I'axe touristique de la "route des crites" faisant le pont entre les zones caractéristiques et fonctionnelles du "Coeur" et "desi hautsi lieux" de l'écomusée. Photo: Pierre Mayrand

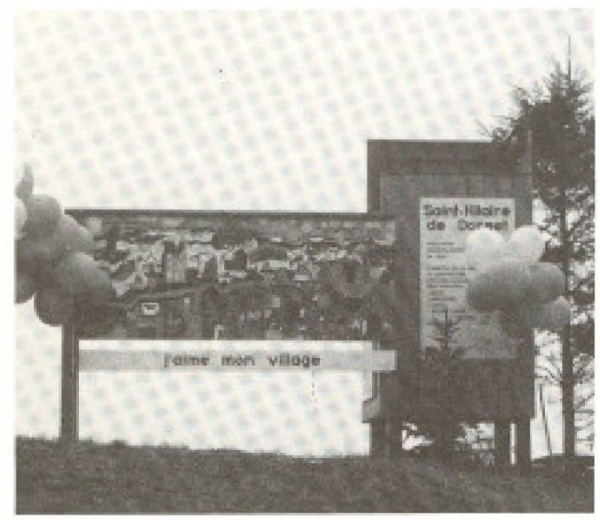

L'exhibit de plein air de Saint Hilaire de Dorset, inauguré en 1980, comme la première antenne du musée "pour tous, par tous". Cette création collective de l'ensemble de la population (230 habitants) est le premier élément constitutif d'un ensemble de réalisations culturelles, parmi les plus denses au Québec en rapport avec le nombre d'habitants: Maison des gens de Saint-Hilaire, Mémoire collective, Comité d'action culturelle et touristique... L'exhibit est un hommage aux pionniers et à l'environnement. Il est un outil de réflexion permanente et de rappel de l'événement écomuséal.

Photo: Pierre Mayrand

${ }^{2} \mathrm{M}$. Pierre Mayrand est Directeur du module Animation et recherches culturelles a I'UOAM. Président fondateur de l'Écomusée de la Haute-Beauce et du Mouvernent international pour une nouvelle muséologie IMINOMI.
Dans ses principes comme dans ses applications, l'ecomusé est directement concerné par la culture et par la région mais n'a généralement que des liens secondaires avec le tourisme. Quoiqu'ici, au Québec, une même finalitế sociale globale a fait naître la chartre du tourisme culturel ${ }^{(1)}$ et le premier écomusée, celui de la Haute-Beauce.

Dans les deux cas, il s'agit de gestes d'affirmation nationale par la voie du développement culturel, une forme de résistance à l'envahissement du Québec par des cultures étrangères largement diffusées par les massmedia et les autres entrepreneurs de l'industrie culturelle

La chartre, s'inspirant du tourisme social. proclame que l'expérience touristique peut être porteuse de culture pour les visiteurs et pour les visités; l'écomusée fụt - et reste une expérience communautaire de développement total ${ }^{(2)} \mathrm{s}^{4}$ articulant sur la recherche d'identité, sur la création et donc, sur la culture vivante.

\section{Écomusée et culture}

Il existe des entreprises de développement régional qui, au-delà des contingences économiques et sociales, se préoccupent du développement par l'intérieur du potentiel d'un groupe ou d'une région. Certains b́comusées ont adopté un modèle systếmatique de développement culturel intégré aux réalités régionales et à leurs dynamismes psychiques. Le musée-territoire de la Haute-Beauce, au Québec, est un de ceuxlà; il expérimente le passage-difficile-d'une conscience prisonnière du passé (le patrimoine héritage) à une appréhension consciente du rôle que chacun peut jouer dans la préparation du devenir collectif (le patrimoine de développement). Le développement culturel est donc un processus qui s'opere dans une collectivité en suivant d'abord ses forces endogènes.

Au point de départ de ce processus se situent les actes de recormaissance: reconnaissance de son territoire, des autres, de ce qui apparente chacun aux autres, des "nous". A cette ćtape, fermée sur elle-même, la communauté découvre ses ressemblances. Puis viendra, généralement, la prise de conscience des différences entre le "nous" et les autres, étape nécessaire à la phase ultérieure, celle des échanges, des interactions avec le reste du monde, celle aussi de la création, de l'invention des formes et des outils de son développement.
Précisons ici - nous y reviendrons - que les opérations touristiques ne peuvent s'entreprendre qu'à l'étape des échanges, quand la collectivité est en mesure d'établir ses interrelations avec l'extérieur.

L'écomusée, en définitive, se caractérise par trois démarches essentielles, intimement liées: I'autodéfinition d'un territoire, la participation des populations à l'élaboration et à la gestion du processus d'autodéveloppement et enfin, l'éclatement des activités sur la totalité d"un territoire dans une perspective de globalité. Le patrimoine est alors l'ensemble des interrelations qui régissent la vie, le dynamisme et l'environnement d'un territoire dont une population devient consciente. Dans les faits, ces principes fondamentaux de l'écomuséologie (Oikos = maison) demeurent le plus souvent des catégories séparées de l'esprit, inventées afin de promouvoir un modèle interdisciplinaire de société. Ils sont rarement menés simultanément aux conclusions ultimes qu'ils annoncent, compte tenu des exigences et de la complexité du processus. Les meilleures intentions sont en effet découragées par des attitudes de professionalisme statique et par un manque de préparation au travail communautaire, notamment à l'éducation populaire.

\section{L'écomuséologie tributaire du contexte social et politique}

Les grandes expériences de prise en charge par le peuple québécois de son propre devenir, à partir des annés ' 60 , le cheminement télescopé qui le conduira de la découverte de soi-même (étape folklorique), à l'affirmation de son identité (étape de contestation et de revendication), à la création des outils de son développement (ćtape technocratique), en passant par la démocratisation culturelle (diffusion, accessibilité), les modèles alternatifs de prise en charge (autogestion, groupes communautaires), le partage des responsabilités (la régionalisation et la décentralisation), se retrouvent dans la constitution de nos écomusếes qui prennent naissance en 1978. Les événements marquants sont le document de travail pour l'evolution de la politique culturelleb et la politique québécoise du développenent culturel ${ }^{[4 !}$.

Coup sur coup naissent, à partir de 1978 . les écomusées de la Haute-Beauce (région de Québec), de la Maison du Fier Monde, de l'Insulaire, de Saint-Constant, des 2-Rives (Région de Montréal), de la Vallée de La Rouge (Laurentides). Ils se regrouperont au sein d"une association, l'Association des éco- 
musées du Québec (1983); ils seront l'hôte d'un colloque international, le premier atelier international "'Écomusées/ nouvelles muséologie" (1984) qui produira la Déclaration de Québec ${ }^{(5)}$ et donnera naissance au Mouvement international pour une nouvelle muséologie, organisation affiliée à l'ICOM. L'Écomusée de la Haute-Beauce sera accrédité par le Ministère des Affaires culturelles, recevra le prix du mérite de l'Association des Musées canadiens (pour la création du premier écomusée populaire en Amérique du Nord) et le prix du développement (La Laurentienne) de la région de Québec en 1985. Son rôle de "développeur" régional sera également reconnu par les sommets économiques de Québec et de l'Estrie.

Le mouvement, inspiré des écomusées français et de ses principaux auteurs ${ }^{(6)}$, est diffusé dans les milieux de Pares Canada qui entretiennent, à la suite de la crise de Forillon, des relations suivies avec l'Écomusée du Creuzot-Montceau-les-Mines en France, solution alternative aux centres culturels issus de la contestation de 1968. Le musée du Quétbec en devenir, concept muséologique ${ }^{71}$, par la définition qu'il donne du musée et par sa référence à Lévi-Strauss, introduira lo doute fondamental sur les missions traditionnelles de l'institution muséologique, reprenant, vingt ans plus tard, les appels lancés du haut de la tribune de l'Unesco par Stanislas Adotévi ${ }^{\left[{ }^{(B)} \text {. }\right.}$.

Le contexte politique - fait à noter - fut sensible à la philosophie que les ćcomusées ont essayé d'incarner. Il semble favoriser maintenant l'innovation et les interprétations permissives du rôle de la culture dans la société contemporaine. Ainsi, peut-on lire cette phrase assez étonnante dans un document diffusé par le Ministère du Conseil exécutif vers 1978: 'La decentrallisation c'est d'abord un acte de confiance envers les individus et un appel à leur créativité. Elle repose essentiellement sur la conviction que les citoyens seront plus en mesure de définir eux-mêmes leurs besoins et de répondre avec originalite et invention à leurs aspirations s'ils exercent une prise directe sur la production des services et des activités qui les affectent quotidiennement. C'est par la prise en charge et l'exercice de responsabilités que peut mieux s'exprimer la créativité. " $\{9$;

Les écomusées, par les rôles et les fonctions qu'ils cherchent à assumer, peuvent se présenter comme des applications fidèles d'une telle politique.

\section{Écomusées et tourisme}

Dans le processus de développement culturel qui caractérise l'approche écomuséologique, au moment ou la collectivité expérimente ses ouvertures au monde et ses échanges avec l'extérieur, elle peut envisager la question du tourisme. Il faut s'attendre toutefois à ce que le tourisme qui pourra se développer dans ce contexte aura plusieurs traits particuliers. Il s'apparentera d'abord davantage au tourisme social qu'au tourisme commercial. L'expérience touristique prin- cipale viendra des relations directes et faceà-face entre les visiteurs et les visités. Dans un musée-territoire, la population est indissociable de ses oeuvres, de ses productions culturelles, de ses services d'accueil, de renseignement et d'animation.

En Haute-Beauce, deux ans à peine après les premières sensibilisations, quand la collection Napoléon Bolduc (un antiquaire de La Guadeloupe) fut acquise et installec dans l'ancien presbytère de Saint-Évariste réaménagé par l'écomusée, les visiteurs commencèrent à arriver. Graduellement, à mesure que d'autres projets se réalisaient sur le territoire, les visiteurs de passage, de même qu'un nombre grandissant de visiteurs spécialisés, venus parfois de l'étranger' (une forme de tourisme de congrès ou de tourisme-"école") s"arrêtèrent davantage en Haute-Beauce. Ceci devint possible grâce à l'originalité des outils de développement et du processus d'implication populaire.

Mais la fréquentation touristique arriva à plafonner (entre 7000 et 8000 visiteurs par année) faute d'infrastructures d'accueil suffisantes. Pour que la fonction touristique puisse s'étendre, il a fallu penser à créer un pôle de développement. Les projets en cours dans la région du Lac Mégantic et du parc Frontenac, sans etre touristique à prime abord, sauront intéresser davantage de visiteurs.

Un de ces projets, le plus important peut-être à l'heure actuelle pour les Hauts-Beaucerons de l'Estrie, est la réalisation de la Maison du Granit dans les Mornes St-Sébastien au Lac Drolet. Cette réalisation qui se situe parfaitement dans le processus de développement culturel du territoire est riche de significations pour la Haute-Beauce. Elle manifeste d'abord que l'aire des échanges s'agrandit puisque la Maison du Granit a reçu l'appui du sommet de l'Estric. Le contenu de ce projet, qui rejoindra davantage les hommes, aidera à l'équilibre et contribuera à l'amalgame de plusieurs catégories de populations dans le développement total (à ses débuts, l'écomusée fut largement un champ d'affirmation des femmes).

L'entrepreneur beauceron trouvera à se manifester et à s'exprimer dans la Maison du Granit et montrera que les affaires et la culture se rejoignent. Dès cette anné, des expositions sur des entrepreneurs se tiendront en divers lieux du territoire. Le programme à cé sujet, d'une durée de cinq ans, culminera en 1992 quand se tiendra à Québec le Congrès Mondial des Musées.

Enfin, avec la Maison du Granit, la Beauce et l'Estrie, déjà associées dans la préparation d'exhibits (Haute-Beauce créatrice en 1982) et dans les échanges avec l'écomusée du Coglais en France, se rapprochent pour former un tandem.

Au plan touristique, la Maison du Granit deviendra le pôle souhaité qui justifiera peutêtre, par la suite, des investissements privés pour l'hébergement, la restauration, les circuits équestres, etc. Le projet regarde également vers les États de la NouvelleAngleterre, bassin potentiel de fréquentation et d'échanges.

L'écomusée ne peut être d'abord une structure du développement touristique. Mais, par son action culturelle, il donnera à une collectivite des occasions de s'ouvrir aux visiteurs étrangers et de les accueillir de façon personnelle et crétive dans le cadre de ses réalisations propres. Ces visiteurs pratiqueront alors, au sens plein du terme, le tourisme culturel. $f$

\section{Refférences}

11) La déclaration quebécoise du tourisme culturel, RONLQ-ATT, Comité de promotion du tourisme culturel, 1978.

(2) Le muste " intégral" (musto intégral), préconisé par la déclaration de Santiago. Chili, en 1972; on "llécodeveloppement (ccodesarollo) mis de I avant par la déclaration d'Oastepec, Mexiqué, en 1985

(3) Pour l'évolution de la politique culturelle, ministère des Affaires culturelles, Jean-Paul Lallier, document de travail, 1976

(4) La politique québicoise du développenent culturel (2 volumes), Le Ministre d'Etat au Developpenent culturel, Ėditeur officiel du Quehes. 1978.

(5) La declaration de Quebec, Premier Atelier International: I'Ecomused/Nouvelle nuscologic, Quebec, octobre 1984.

(6) Henri-Georges Riviere et Hugues de Varine. Ils sont venus au Québec plusicurs fois depuis 10 ans.

(7) Le musbe du Québec en devenir; concept musciologique, ministere des Affaires culturelles, Muse du Qué bec, axout 1979 .

(8) ADOTEVI, Stanislas, Le wusét dans les systèmes diducarifs ef culrurels conemporains ", in Le nuste au service des hommes, aujourd'hui et demain, Actes de la Oe conférence générale de l'ICOM, Paris, Maison de I'UNESCO, 1972.

(9) La décentralisation, une perspective communantaire gouvelle. Fascicule I: Une vue d'ensemble, ministère du Conscil exécutif, Sectétarias a I'aménagement et à la decentralisation. sans date (vers 1978). 